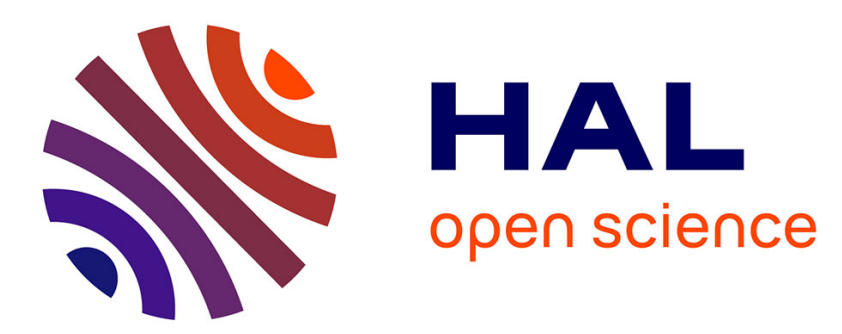

\title{
INEDUC : focales sur les inégalités scolaires, de loisirs et de pratiques numériques chez les adolescents
}

\author{
Pascal Plantard, Mickaël Le Mentec
}

\section{To cite this version:}

Pascal Plantard, Mickaël Le Mentec. INEDUC: focales sur les inégalités scolaires, de loisirs et de pratiques numériques chez les adolescents. Terminal. Technologie de l'information, culture \& société, 2013, Les TIC à l'école, 113-114, pp.79-91. hal-01084282

\section{HAL Id: hal-01084282 \\ https://hal.science/hal-01084282}

Submitted on 16 Jun 2015

HAL is a multi-disciplinary open access archive for the deposit and dissemination of scientific research documents, whether they are published or not. The documents may come from teaching and research institutions in France or abroad, or from public or private research centers.
L'archive ouverte pluridisciplinaire HAL, est destinée au dépôt et à la diffusion de documents scientifiques de niveau recherche, publiés ou non, émanant des établissements d'enseignement et de recherche français ou étrangers, des laboratoires publics ou privés.

$$
\text { Copyright }
$$




\title{
INEDUC : focales sur les inégalités scolaires, de loisirs et de pratiques numé- riques chez les adolescents
}

\author{
Pascal Plantard et Mickaël Le Mentec*
}

\section{Introduction}

$\mathrm{N}$

otre consortium pluridisciplinaire (sciences de l'éducation, géographie sociale, anthropologie et sociologie) composé de plusieurs équipes des universités Rennes 2, Caen, Angers et du GIS Marsouin ${ }^{1}$ a obtenu un contrat de recherche $\mathrm{ANR}^{2}$ à propos des inégalités éducatives, qui présente la caractéristique de croiser les parcours scolaires avec les pratiques numériques et de loisirs des adolescents.

Ce projet, INEDUC, vise à identifier les inégalités éducatives liées aux contextes et espaces de vie des jeunes âgés de 11 à 15 ans (période scolaire du collège) en France. L'éducation renvoie ici aux acquisitions qui résultent des interactions entre l'individu et son environnement physique et humain, englobant tant les transmissions par action volontaire que par imprégnation.

L'objectif est d'analyser différents contextes qui influencent les parcours éducatifs des jeunes : celui de l'institution scolaire, celui des loisirs sportifs et culturels et celui, transversal aux deux précédents, des environnements numériques. Ces contextes, par les types d'usages des ressources localisées qu'elles déterminent, induisent des différences dans les parcours sociaux des adolescents. Faute d'actions préventives ou réparatrices efficaces, ces différences se traduisent en inégalités qui, cumulées dans l'espace et

\footnotetext{
* Université Rennes 2 - CREAD et GIS MARSOUIN. Pascal.Plantard@uhb.fr et mickael.lementec@uhb.fr 1 Le Groupement d'Intérêt Scientifique M@rsouin, créé en 2002 à l'initiative du Conseil régional de Bretagne, fédère 11 centres de recherche en sciences humaines et sociales travaillant sur les usages des technologies de l'information et de la communication.

2 L'Agence Nationale de la Recherche est un établissement public français à caractère administratif créé en 2005. L'ANR finance les équipes de recherches publiques ou privées sous la forme de contrats de recherche obtenus en répondant à des appels à projets.
} 
dans le temps chez une partie des jeunes, peuvent mettre en péril la cohésion sociale. Identifier les différents mécanismes de la construction de ces inégalités et leurs liens peut fournir des outils théoriques utiles pour la promotion de l'épanouissement du potentiel de chaque jeune afin qu'il puisse devenir acteur de son propre développement et du développement social. La dimension comparative de ce projet, qui traitera, sur le plan quantitatif, de trois territoires régionaux (Aquitaine, Basse-Normandie et Bretagne) et sur le plan qualitatif, d'espaces locaux sélectionnés dans ces régions, vise à enrichir la connaissance sur la diversité des politiques d'éducation et plus globalement sur les types d'effets de lieu sur les parcours sociaux et éducatifs des jeunes.

Relier les trajectoires scolaires, les pratiques de loisirs et les pratiques numériques aux caractéristiques propres au jeune, à sa famille, à son environnement ou à son mode de vie, nous permettra d'analyser les « fractures éducatives ", soit les différences de niveaux de connaissances et d'éducation qui distinguent les adolescents.

La réflexion sur la construction des parcours paraît particulièrement pertinente sur la tranche 11-15 ans car cette période d'adolescence-jeunesse est déterminante et moins investiguée que la jeunesse de 16 à 25 ans. En effet, à cet âge, les adolescents prennent une réelle autonomie d'action vis-àvis de la famille et sont moins assujettis aux choix parentaux et aux activités familiales.

Au moment de l'entrée au collège, ils conquièrent et explorent de nouveaux espaces hors de la famille et hors des établissements scolaires : espaces du quartier ou de l'agglomération, espaces publics et équipements socioculturels et sportifs, espaces numériques (réseaux sociaux, sites commerciaux, ludiques ou d'information) en y déployant des activités moins contrôlées par les adultes. De ce fait, il s'agit véritablement d'une période cruciale de construction du parcours éducatif et de construction identitaire incluant l'acquisition de schèmes d'action, de pensée et de perception déterminants.

En accord avec les autres équipes du programme INEDUC, notre article est porté par l'équipe CREAD 3 -M@rsouin. Il est centré sur la problématique des inégalités d'usages du numérique par les collégiens. Il fera la synthèse de l'état de l'art et des recherches quantitatives sur ce sujet et se poursuivra par des propositions théoriques permettant de renouveler la conceptualisation de la problématique des TIC à l'école.

\section{Panorama européen des enquêtes quantitatives concernant les TICE}

Notre programme de recherche a démarré en janvier 2012 par :

- un état de l'art des travaux francophones sur cette problématique ;

3 Centre de Recherche sur l'Éducation, les Apprentissages et la Didactique.

80

] terminal $\mathrm{n}^{\circ} 113$ [

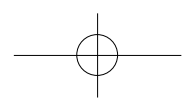


- une recherche d'éléments quantitatifs d'appui à la construction d'un questionnaire destiné à 4000 collégiens complété par un questionnaire «parents ».

\section{Instantané sur l'équipement numérique des collèges}

L'enquête ETIC ${ }^{4}$ (2010) montre que les taux d'équipements technologiques à usage éducatif sont très nuancés en fonction des académies. Le nombre d'élèves par ordinateur peut varier de 1 pour 3,9 collégiens jusqu'à 1 pour 13 selon les territoires : en moyenne, on compte 5,6 collégiens par ordinateur. Ce ratio s'élève à 9,5 lorsqu'on prend en compte les équipements de moins de cinq ans.

En termes d'accès à l'Internet, ETIC montre que le haut débit n'est pas encore généralisé dans tous les établissements scolaires français. Cela a des incidences sur les usages développés. A peine plus de la moitié des collèges a accès à un débit supérieur à 2 Mo, ce qui constitue, selon les enquêteurs, le débit minimum pour développer des usages pédagogiques confortables (usages de la vidéo, de l'audio ou des espaces numériques de travail). Parmi eux, seulement $16 \%$ des collèges possèdent un débit supérieur à 10 Mo.

Les services en ligne mis à disposition sont également très nuancés. Près de $80 \%$ des collèges mettent en ligne des informations concernant l'actualité de l'établissement, $64 \%$ des documents et des ressources pédagogiques à destination des élèves. Depuis 2008, les collégiens ont l'obligation de posséder le $\mathrm{B} 2 \mathrm{i}^{5}$ pour obtenir le brevet des collèges. L'enquête a cherché à obtenir le pourcentage d'élève de quatrième ayant acquis, au début de l'année, $40 \%$ des modules du $\mathrm{B} 2 \mathrm{i}$. Ce résultat s'élève à $22,8 \%$.

\section{L'ordinateur multitâche ou l'objet incontournable des adolescents}

En lien avec le panorama sur l'équipement des collégiens, l'enquête de 2010 sur l'enfance des loisirs [octobre et al., 2010] montre que lors du passage en quatrième, $20 \%$ des adolescents possèdent un ordinateur personnel. L'ordinateur prend une place de plus en plus importante avec l'avancée en âge car, s'ils ne sont que $14,5 \%$ à en avoir une pratique quotidienne à 11 ans, ils sont $69 \%$ à l'utiliser tous les jours à 17 ans.

Cette place que prend le numérique dans la vie des adolescents s'explique par le fait que l'ordinateur permet de développer des usages très diversifiés : écouter de la musique, communiquer, regarder une série ou un film, faire des recherches sur Internet ou encore jouer aux jeux en ligne. A ce titre,

4. L'Enquête sur les Technologies de I'Information et de la Communication (ETIC) est une enquête nationale destinée à fournir des indicateurs sur les TICE dans les écoles, collèges et lycées publics au niveau de l'équipement, de l'infrastructure, des moyens humains, des services numériques, de la sécurité des mineurs face à internet, de la formation des enseignants et plus globalement de tous les aspects TIC qui font l'objet d'une priorité ministérielle.

5. B2i : Brevet informatique et internet. Ce brevet permet de valider les compétences des élèves, grâce à plusieurs modules, autour des usages de l'internet de l'informatique.

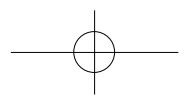


$58,5 \%$ des grands adolescents 6 ont déclaré aux enquêteurs développer plus de cinq activités différentes autour de l'ordinateur.

Par ailleurs, $73 \%$ des jeunes qui sont en début de collège jouent principalement aux jeux vidéo. Les usages communicationnels ou la consommation de produits culturels deviennent plus fréquents avec l'avancée en âge. Les recherches sur internet en lien avec le travail scolaire s'intensifient également au fur et à mesure que les adolescents grandissent. A 17 ans, l'ordinateur est l'outil privilégié qui permet de développer à la fois des usages culturels, communicationnels et de travail. Octobre et al. [2010] constatent qu'avec l'avancée en âge, les adolescents passent du statut de consommateurs passifs qui regardent la télévision, à celui d'usagers actifs qui choisissent leurs activités et déposent du contenu sur Internet grâce aux usages qu'ils ont de l'ordinateur.

\section{Un recul de la télévision avec l'âge}

Octobre et al. [2010] montrent aussi que l'audience télévisuelle baisse significativement avec l'avancée en âge. Les jeunes de 11 ans sont 55,5\% à la regarder le mercredi après-midi et un peu moins de $50 \%$ le dimanche matin ou juste après l'école. Ils la regardent le soir uniquement pendant le week-end car les parents établissent des règles de coucher en semaine qui restreignent cette pratique.

Plus on avance dans l'âge, plus les calendriers s'inversent, c'est-à-dire que les grands adolescents sont 66,5\% à la regarder le soir en semaine alors que, contrairement aux plus jeunes, ils ne sont que 3,5\% à la regarder le mercredi après-midi. Les contenus regardés diffèrent également en fonction de l'âge : les dessins animés laissent progressivement la place aux séries télévisées diffusées en soirée. Cette concurrence entre la télévision et la pratique active de l'ordinateur et d'internet par les adolescents avait été aussi repérée par Olivier Donnat dans son enquête sur les pratiques culturelles des français à l'ère du numérique [2008].

\section{Un usage central : l'écoute de la musique enregistrée}

L'écoute de la musique enregistrée prend une place de plus en plus importante avec l'avancée en âge. Selon Octobre et al. [2010] les modalités d'écoute informent à la fois sur le mode d'individualisation caractéristique de cette période $(94,5 \%$ des adolescents âgés de 17 ans écoutent de la musique seuls) mais aussi sur celui de la sociabilité amicale puisque $40 \%$ d'entre eux partagent de la musique avec leurs amis. Ce résultat témoigne du rôle que joue la socialisation amicale dans la découverte de nouveaux artistes,

6. Les chercheurs de l'enquête " l'enfance des loisirs " considèrent les grands adolescents ceux qui sont âgés de 17 ans.

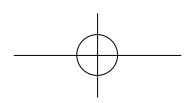


mais aussi dans la construction des goûts et des préférences. Dans ce contexte, les agents de socialisation, en particulier les amis, jouent un rôle essentiel dans l'accès aux objets culturels, la transmission de contenus ainsi que le développement des goûts et préférences personnels.

Au fur et à mesure que l'on avance dans l'âge, les amis deviennent de plus en plus prescripteurs et accompagnateurs dans les pratiques des adolescents, et ce de façon collective. La socialisation amicale supplante celle de la famille (parents, frères et sœurs) et celle de l'école dans les activités de découverte et d'échange de contenus.

Ainsi, par leurs pratiques numériques, les adolescents accèdent à des ressources culturelles et ont la possibilité de choisir, rejeter, agencer ces différents contenus afin de développer leurs propres sensibilités au contact des instances de socialisation (famille, école, copains). Il est important de préciser que plus les adolescents grandissent, plus les goûts sont variés.

\section{Les usages des jeunes européens et la sécurité en ligne}

Financée par le programme de la Commission européenne "Safer internet », l'enquête EU Kids Online ${ }^{7}$ traite de la sécurité et de la navigation en ligne des jeunes de 9 à 16 ans. Cette enquête internationale s'appuie sur un échantillon de 25142 jeunes, ainsi que leurs parents, dans 25 pays européens ${ }^{8}$.

$93 \%$ des jeunes interrogés au cours du printemps et de l'été 2010, vont en ligne au moins une fois par semaine. Ils sont $60 \%$ à le faire tous les jours. Les enfants se connectent de plus en plus jeunes : la pratique régulière d'internet commence à 7 ans au Danemark et en Suède. Les usages d'Internet sont très variés en Lituanie, République Tchèque, Estonie, France et Suède. Ils sont plus restreints en Irlande et en Turquie.

L'enquête montre que $12 \%$ des jeunes âgés de 9 à 16 ans disent avoir été tracassés par quelque chose sur Internet. C'est le cas par exemple de celles et ceux qui se sont sentis harcelés par des messages agressifs. Dans un cas sur vingt, cela génère davantage de souffrance qu'une simple blessure. Il est important de noter que les risques augmentent avec l'âge, puisque $14 \%$ des 9-10 ans ont été confronté au moins à un risque, alors qu'ils sont $63 \%$ des 15-16 ans. Parmi ces risques, l'enquête relève l'exposition à des images sexuelles ou pornographiques dont internet est devenu l'une des principales sources de diffusion. Parmi celles et ceux qui ont été exposé à ce type de contenu, $53 \%$ en ont parlé à quelqu'un lorsque cela est arrivé, notamment

7. Le réseau EU Kids Online a été coordonné par la London School of Economics avec des équipes de recherche propres dans chacun des 25 pays sous le contrôle d'un comité d'experts internationaux : www.eukidsonline.net

8. Allemagne, Autriche, Belgique, Bulgarie, Chypre, Danemark, Espagne, Estonie, Finlande, France, Grèce, Hongrie, Irlande, Italie, Lituanie, Norvège, Pays Bas, Pologne, Portugal, République Tchèque Roumanie, Slovénie, Suède, Turquie et Royaume Uni.

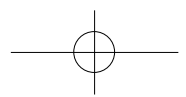


$25 \%$ à un de leurs parents. La communication en ligne avec des inconnus est un des risques les plus répandus : $30 \%$ des 9-16 ans déclarent avoir communiqué sur internet avec une personne qu'ils ne connaissaient.

EU Kids Online démontre que les parents ne sont pas toujours au courant du fait que leurs enfants aient déjà rencontré un risque sur internet. $40 \%$ des parents dont les enfants ont déjà été confrontés à des images de type sexuel pensent que cela ne leur est pas arrivé. Cela traduit bien un manque de sensibilisation des parents autour des questions de sécurité sur internet. Néanmoins, un peu plus de la moitié des parents restent à côté de leurs enfants lorsqu'ils naviguent sur internet, et près des trois quarts discutent avec eux des usages pratiqués. Ils sont aussi conseillers auprès de leurs enfants à hauteur de $50 \%$, notamment sur la manière de se comporter en ligne avec les autres.

Enfin, l'utilisation par les parents de dispositifs de sécurité permettant par exemple de restreindre l'accès en bloquant, filtrant ou en observant l'historique est relativement faible ( $28 \%$ ). Il est important de noter qu'en ce qui concerne les conseils pour la sécurité en ligne, les jeunes considèrent qu'ils sont le mieux conseillés d'abord par leurs parents à $63 \%$, ensuite par leurs enseignants à $58 \%$ et enfin par les groupes de pairs à $44 \%$.

\section{Les « fractures numériques 》}

À notre connaissance, il n'y a pas d'études quantitatives, en langue française, traitant spécifiquement des inégalités d'accès ou d'usages du numérique par les jeunes de 11 à 15 ans. Néanmoins, plusieurs recherches traitent des inégalités numériques dans les familles, ce qui nous paraît très précieux pour contextualiser les inégalités des enfants. L'étude des microdonnées 2008 des enquêtes annuelles Eurostat-OCDE ${ }^{9}$ de diffusion des TIC au sein des ménages dans vingt pays européens (79 000 ménages, 166000 personnes) et en Corée du Sud (17 000 ménages, 41644 personnes) démontre que les variables socio-économiques permettent de prédire significativement l'accès, l'usage et la diversité des usages d'internet.

Ces variables sont, par ordre d'importance : la localisation géographique, l'âge, le niveau d'éducation, le genre, la présence d'enfant et le revenu. La dimension culturelle est très importante. Par exemple, en Corée, c'est la composition de la famille qui prime et les activités de loisirs en ligne sont effectuées hors domicile. Le communiqué Eurostat du 13 décembre 2011 précise que dans l'Union Européenne des 27, « près d'un quart des personnes de 16 à 74 ans n'a jamais utilisé Internet ${ }{ }^{10}$. Celui du 26 mars 2012 nous

\footnotetext{
9. Étude présentée par Pierre Montagnier de l'OCDE au forum des usages coopératifs de Brest en juillet 2010, consulté le 24 mai 2013, disponible sur www.a-brest.net/article6090.html

10. " Accès à Internet et utilisation des TIC en entreprise en 2011 ", consulté le 24 mai 2013, disponible sur http://epp.eurostat.ec.europa.eu/portal/page/portal/information_society/publications
}

84 ] terminal $n^{\circ} 113[$

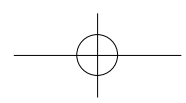


signale qu'il reste encore un quart de non-internautes en Europe ${ }^{11}$. Effectuée annuellement depuis 2003 auprès d'un échantillon de 2000 familles, l'enquête Particuliers de M@rsouin démontre que les déterminants socio-économiques permettent de prédire à $80 \%$ les comportements numériques. Il reste donc un seuil de $20 \%$ qui ne répond pas à ces déterminants et qui ouvre des pistes de recherches sur les inégalités du coté des imaginaires, des représentations et du fonctionnement psychique individuel.

\section{De la fracture numérique à l'e-inclusion}

Lors de l'enquête M@rsouin 2009, le facteur isolement devient « l'indicateur étrange " de l'exclusion numérique, faisant sauter les catégories sociologiques classiques (âge, revenu, capital culturel). $65 \%$ des personnes interrogées invoquent l'isolement comme premier facteur du non-usage du numérique. Au cœur des phénomènes d'exclusion numérique, on trouve donc la notion d'isolement social qui combine faiblement les questions d'âge (les seniors isolés) et les travailleurs pauvres mais très fortement les populations qui n'ont que les minimas sociaux pour vivre.

Il serait hâtif d'en déduire que la pauvreté renvoie à une forme unique d'isolement qui conditionnerait l'exclusion numérique. C'est le «sentiment » d'isolement qui importe. Cette catégorie comprend aussi des personnes qui n'ont pas nécessairement de problème d'argent mais qui se retrouvent dans des situations de vie qui les isolent.

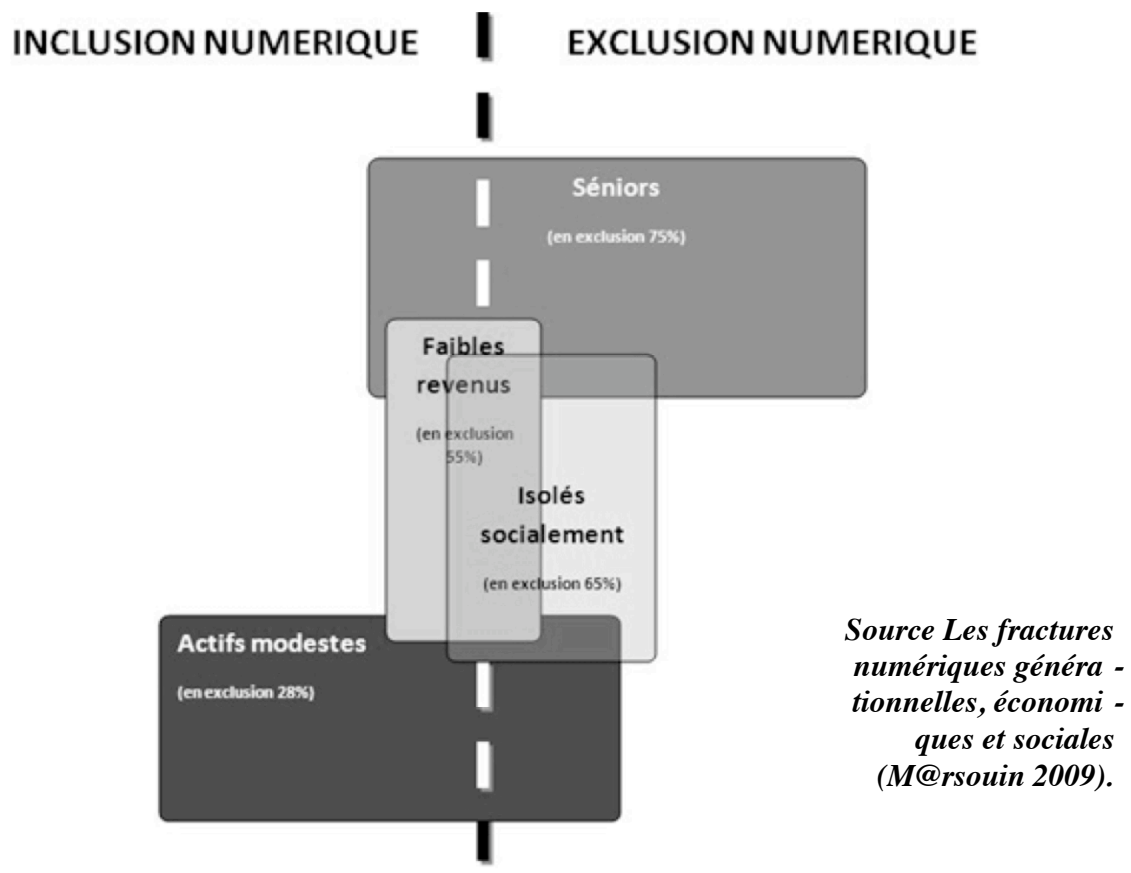

11. Les compétences numériques dans I'UE27 en chiffres, consulté le 24 mai 2013, disponible sur http://epp.eurostat.ec.europa.eu/cache/ITY_PUBLIC/4-26032012-AP/FR/4-26032012-AP-FR.PDF

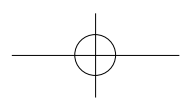


La question de l'absence de temps disponible à consacrer au numérique est souvent évoquée comme facteur de non-usage par les personnes qui se sentent isolées, en particulier par une population bien identifiable : les familles monoparentales qui sont très souvent portées par des femmes. La « fracture numérique ", même déclinée au pluriel, n’est pas un concept opératoire. Elle n'est pas une simple conséquence des inégalités sociales et économiques préexistantes. Elle est plutôt le produit de la rencontre singulière de chaque personne avec les cultures numériques.

Cette rencontre, en fonction des histoires singulières, favorisera ou non le désir, l'envie, la motivation pour se lancer dans les usages des Tic. Au-delà des questions d'âge, de niveau d'éducation et de revenus, c'est une réflexion sur la capacité à développer des relations sociales dans un environnement qu'il faut privilégier aujourd'hui pour lutter contre les exclusions numériques et faire advenir une nouvelle forme de société de l'information non plus excluante mais inclusive. C'est dans le cadre théorique de l'e-inclusion, définie comme l'étude conjointe des nouvelles exclusions générées par les TiC, mais aussi des nouvelles potentialités d'inclusion (éducation, médiation, formation) issues des usages du numérique que nous analyserons les données empiriques du projet INEDUC.

\section{Les concepts clés d'INEDUC}

Un travail d'exploration conceptuelle a été mené par l'équipe INEDUC afin de préparer un modèle transdisciplinaire d'analyse des données empiriques récoltées. Nous vous livrons trois des nombreuses pistes que nous avons empruntées sur les chemins du numérique.

* De l'environnement à la culture numérique

L'environnement numérique est un espace structuré par des instruments technologiques divers, permettant aux usagers d'accéder à des ressources et à des services numériques présents sur les machines ou en ligne.

Le premier élément d'un environnement numérique est très souvent l'ordinateur auquel on associe différents périphériques plus ou moins spécialisés (imprimante, scanner, disques de stockage, appareil photo numérique, webcam, robots, lunettes 3D). Dans l'école, l'environnement numérique est aussi étroitement lié à une multitude d'expressions telles que l'espace numérique de travail, le bureau virtuel ou encore la plate-forme d'apprentissage en ligne. Au sens institutionnel du terme, les environnements numériques désignent les espaces en ligne qui permettent aux personnes (élèves, enseignants, étudiants, personnels, parents) d'accéder aux ressources et aux outils mis à disposition par une institution ou un établissement.

Le numérique n'est pas réductible à ses environnements, c'est aussi une véritable culture. Au delà du processus informatique de numérisation

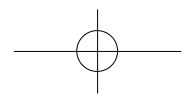


(digitalisation), les Tic ont fait naître une nouvelle civilisation numérique. Les usages des instruments technologiques modifient en profondeur la communication, le rapport au savoir et les liens sociaux. Cela percute autant nos organisations, en particulier l'école, que nos imaginaires socio-historiques. Comme l'écrit Doueihi "L'humanisme numérique... est le résultat d'une convergence entre notre héritage culturel complexe et une technique devenue un lieu de sociabilité sans précédent » [2011, p. 9].

Il illustre cet humanisme numérique par les nouvelles pratiques « amicales » qui ont cours sur les réseaux sociaux numériques et qui nous intéressent tout particulièrement du point de vue de la construction identitaire des adolescents : "Peut-on ou doit-on tout partager avec son ami, avec ses amis ? Peut-on ou doit-on abandonner toute propriété individuelle au profit de la collectivité ? Ou bien faut-il préserver la propriété individuelle et la soumet tre au choix de l'ami ? Derrière ces questions on retrouve les problèmes qui sont en grande partie les nôtres aujourd'hui dans la culture numérique: quels sont les liens entre le partage, l'amitié numérique et le domaine public ? Quelles sont les formes d'échange et l'économie qui les soutient dans le monde de la sociabilité numérique? Est-ce que l'amitié inaugure une nouvelle ère, avec un nouveau paradigme de la propriété et de la valorisation? Ou bien s'agit-il tout simplement d'une exploitation économique classique de la mise en forme numérique de l'amitié et de ses extensions par les plates-formes actuelles? 》 [2011, p. 88].

Pour se poser de telles questions, il faut reconnaître que les usages du numérique s'installent sur des histoires et des territoires culturels, économiques et sociaux. Pour INEDUC, méthodologiquement, les parcours d'usages du numérique des adolescents seront mis en perspectives par les parcours scolaires et de loisirs autant qu'ils les mettront en lumière.

* De la pratique à l'usage

Opposée à la théorie abstraite, la pratique qualifie l'activité humaine concrète. Indissociable l'une de l'autre, la théorie et la pratique fonde l'une des dialectiques fondamentales des sciences modernes. La pratique ne peut se définir qu'à partir de l'adjectif qui la qualifie. Ainsi, certains chercheurs définissent les notions de pratiques socialisées [Plantard, 2011], de pratiques culturelles [Coulangeon, 2005 ; Donnat, 2008] en tant que couple, c'est-àdire sans dissociation des deux termes. La pratique témoigne de l'activité concrète de l'individu qui lui permet d'atteindre un objectif.

Elle renvoie à la notion de praxis, d'origine grecque, qui qualifie une pratique humaine structurée par une idée et qui tend vers un résultat concret.

La pratique n'est jamais neutre, elle révèle autant de soi que du monde. Pour l'observer, il est nécessaire de la resituer dans son contexte géographique, social ou encore politique. Pour les pratiques numériques, on observe un processus de socialisation qui s'installe au moment où les individus s'approprient les technologies pour développer des usages [Breton, Proulx, 2002] 
qui, de par leur massification dans le corps social, constituent de nouvelles normes [Plantard, 2011].

Pour construire des outils méthodologiques adaptés à l'observation des pratiques réelles et à leur traduction en usages, nous nous sommes appuyés sur plusieurs enquêtes. Parmi elles, le travail d'Octobre et al. [2010] sur l'enfance des loisirs nous a particulièrement éclairé. L'originalité de cette recherche réside dans le fait que les chercheurs ont interrogé les mêmes individus à différentes périodes de leur adolescence (soit à 11, 13, 15 et 17 ans).

Ce choix méthodologique permet ainsi d'observer l'évolution des pratiques culturelles et numériques en fonction de leur avancée en âge et de saisir les rythmes de progression et de transition, ainsi que les effets de l'origine sociale et du genre. Les chercheurs rendent ainsi comptent des goûts, des préférences et des choix culturels des adolescents au delà de l'héritage parental.

Nous émettons l'hypothèse que les usages des Tic sont en partie responsables de ces changements de comportement, notamment parce que l'usage comporte une dimension créative [De Certeau, 1980 ; Plantard, 2011] qui permet aux individus de mobiliser la technique et de la détourner de leur utilisation prescrite pour répondre à des besoins personnels. Il existe dans l'usage une part d'affranchissement qui favorise l'autonomie, la prise en charge personnelle et le développement de pratiques d'empowerment [Le Mentec, 2010].

* Le terrorisme de l'impact

En juillet 2006, la London School of Economics publie une étude ${ }^{12}$ dans laquelle Stephen Machin, Sandra Mc Nally et Olmo Silva écrivent : "Nous avons la preuve d'un impact positif de l'investissement en TIC sur les performances scolaires dans l'enseignement primaire ".

En mars 2007, le prestigieux "Institute of education center 》 américain livrait une étude ${ }^{13}$ au Congrès qui arrivait aux mêmes conclusions. Enfin, le rapport Fourgous remis au Premier ministre le 15 février 2010, s'appuie sur plusieurs études françaises qui démontrent l'impact positif des TicE sur l'enseignement et l'apprentissage pour proposer 70 mesures pour faire «basculer l'école dans l'ère numérique ».

Nous pourrions continuer cette liste " d'études d'impact » à l'infini à l'image du colloque scientifique international ${ }^{14}$ sur les TIC en éducation qui s'est déroulé les 3 et 4 mai 2012 à Montréal. Dans son programme, on retrouve 67 fois le terme « impact » et 25 fois son pluriel « impacts».

\footnotetext{
12. "New Technology in Schools: Is There a Payoff ? ", consulté le 24 mai, disponible sur http:// ftp.iza.org/dp2234.pdf

13. " Effectiveness of Reading and Mathematics Software Products : Finding from the First Student Cohort ", consulté le 24 mai 2013, disponible sur www.mathematica-mpr.com/publications/pdfs/ effectread.pdf

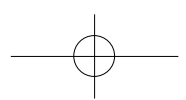


Lorsqu'on évoque les Tic à l'école, nous nous contentons trop souvent de mesurer sans expliquer ni comprendre. Comme l'écrit Alain Gras : « $L a$ fascination pour la mesure, que représente l'évaluation quantitative... occulte le fait que le choix des éléments à mesurer est subjectif et qu'en aucun cas la scientificité ne vient directement de l'objet. " [2010, p. 285]. À l'ère numérique, en éducation comme ailleurs, il est très difficile de s'extraire de la logique de la diffusion mécanique qui voudrait que les industriels inventent des produits que nous consommons sans réfléchir, et de l'image balistique de l'impact. À l'ère numérique, ces causalités simplistes sont portées par des notions comme l'innovation, la fracture numérique, la génération Y... qui justifient toutes les mesures d'impact. Pour sortir de ce terrorisme de l'impact, il convient de considérer le numérique comme :

-Une culture complexe saturée d'idéologies et d'imaginaires [Simondon, 1958 ; Musso, 2009] ;

४Un fait social total qui dessine les phénomènes sociaux et leurs relations [Balandier, 1961] ;

४Un Pharmakon qui désigne, selon Stiegler (2008), l'ambivalence des technologies numériques ;

-Une culture basée sur les techno-imaginaires où l'imaginaire est fortement dépendant de la technique [Musso, 2009].

Au delà de la contextualisation statistique, c'est donc en considérant le numérique comme culture et en se centrant sur l'observation qualitative des pratiques numériques que nous allons aborder INEDUC.

\section{Perspectives de recherche}

Dans le cadre de ce projet, nous avons fait le choix d'interroger et d'observer ces jeunes âgés de 11 à 15 ans en tant que jeunes, et non uniquement en tant que collégiens. Nous avons aussi choisi de ne pas mener notre enquête uniquement dans les collèges mais aussi dans les structures de loisirs et dans les espaces publics numériques. La différence est importante car ces changements de posture ont des effets sur l'observation des pratiques des jeunes et donc sur la qualité du matériel empirique récolté.

Dans INEDUC, nous allons chercher, par l'observation empirique des pratiques effectives du numérique par les collégiens, à comprendre ce qui fait civilisation lorsque les techno-imaginaires initient des pratiques numériques qui se sédimentent en usages. Nous définissons les usages comme des ensembles de pratiques socialisées [Plantard, 2011]. Cette formule stabilisée sert d'appui au travail collectif. Le terme « ensemble » suggère des questions de seuil, de groupes sociaux, de frontières. Les usages fondent de nouvelles normes autour desquelles se créent les sociabilités. Les millions de connexions à Wikipédia ou à Facebook témoignent d'usages sociaux instal-

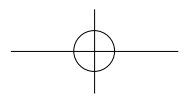


lés chez les adolescents. À l'inverse, malgré les discours ambiants sur l'arrivée massive et imminente des mondes virtuels, Second Life n'a trouvé, en comparaison, qu'un public relativement restreint chez les adolescents. L'adjectif " socialisées » renvoie donc à des questions de constructions collectives et à l'étude des processus d'adoption des normes culturelles, ce qui nous conduit à replacer les usages des TIC dans les contextes socio-historiques. Les différents résultats d'enquêtes nous permettent d'avoir des tendances sur les usages des adolescents et des jeunes, et d'identifier les problématiques que ceux-ci soulèvent.

Il faut noter que les usages autour du réseau social Facebook apparaissent peu car ce réseau ne s'est développé qu'à partir de 2008, en Europe. Or il est aujourd'hui investi de façon massive par les jeunes qui y trouvent un endroit pour développer leur sociabilité, déposer et échanger du contenu (photos, vidéos, musique), écrire et commenter, structurer leur identité numérique et sociale à travers ce qu'ils vont mettre en avant ou obscurcir de leur personnalité [Cardon, 2010]. Nous émettons l'hypothèse que ces usages participent à la structuration de leur identité sociale et ont des effets sur la construction de leur personne, en tant qu'être social.

Il est par ailleurs primordial de repérer si les usages qu'ils font des technologies numériques favorisent leur capacité d'agir et le développement de pratiques d'empowerment, pour celles et ceux qui éprouvent des difficultés d'ordre scolaires et/ou sociales. L'empowerment se traduit ici par une appropriation du pouvoir [Ninacs, 2003], c'est-à-dire par la capacité à mobiliser et à utiliser des ressources (numériques dans ce cas) pour renforcer son action sur l'environnement et contourner ce qui constitue pour eux un obstacle au changement désiré [Le Bossé, 2008].

Dans ce contexte, avec INEDUC, nous chercherons à comprendre comment les usages du numérique, dans leurs dimensions coopératives, sociales et créatrices, participent à la réussite scolaire des 11-15 ans. 


\section{REFERENCES}

BALANDIER, G. (1961). " Phénomènes sociaux totaux et dynamique sociale " in Cahiers Internationaux de Sociologie, volume 30, Paris : PUF, pp. 23-34.

BRETON, P., PROULX S. (2002). L'explosion de la communication à l'aube du XXIe siècle, Paris, La Découverte.

CARDON, D. (2010). La démocratie Internet. Promesse et limites, Paris, Seuil.

COULANGEON, P. (2005). Sociologie des pratiques culturelles, Paris, La Découverte.

DE CERTEAU, M. (1990). L'invention du quotidien, T.1, Arts de faire, Paris, Gallimard.

DONNAT, 0. (2008). Les pratiques culturelles des français à l'ère du numérique, Paris, La Découverte.

DOUEIHI, M. (2011). Pour un humanisme numérique, Paris, Seuil.

GRAS, A. (2010). " L'évaluation d'un fait technique, une métaphysique pour l'hyper sauvage contemporain " in BALANDIER G. (Dir) "Ce qu'évaluer voudrait dire ", Cahiers internationaux de sociologie, Vol CXXVIII-CXXIX, Paris, PUF, pp. 285-298.

LE BOSSÉ, Y. (2008). « L'empowerment: de quel pouvoir s'agit-il ? Changer le monde (le petit et le grand) au quotidien ", in Nouvelles pratiques sociales, Échos et débats, vol. 21, n 1, p. 137-149.

LE MENTEC, M. (2010). "Usages des TIC et pratiques d'empowerment des personnes en situation de disqualification sociale dans les EPN bretons ", thèse de doctorat en sciences de l'éducation sous la direction de Paul Taylor, université Rennes 2 (en ligne, consulté le 24 mai 2013), disponible sur www.marsouin.org/IMG/pdf/These_MIM.pdf

MUSSO P., (2009). " Usages et imaginaires des TIC : la fiction des frictions ", in LICOPPE C. (Dir par), L'évolution des cultures numériques, de la mutation du lien social à l'organisation du travail, Limoges, Fyp Editions, pp. 201-210.

NINACS, W. A. (2003). L'empowerment et l'intervention sociale, Centre de documentation sur l'éducation des adultes et la condition féminine (CDEACF).

OCTOBRE, S., DÉTREZ, C., MERCKLÉ, P., BERTHOMIER, N. (2010). L'enfance des loisirs Trajectoires communes et parcours individuels de la fin de l'enfance à la grande adoles cence, Paris, La Découverte.

PLANTARD, P. (dir). (2011). Pour en finir avec la fracture numérique, Limoges, FYP Editions.

SIMONDON, G. (1958). Du mode d'existence des objets techniques, Paris, Aubier.

STIEGLER, B. (2008). Prendre soin, de la jeunesse et des générations, Paris, Flammarion. 
Partie2-T113 2/08/13 19:08 Page 92

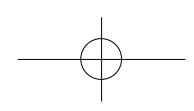

$92 \quad]$ terminal $n^{\circ} 113[$

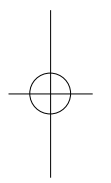

]
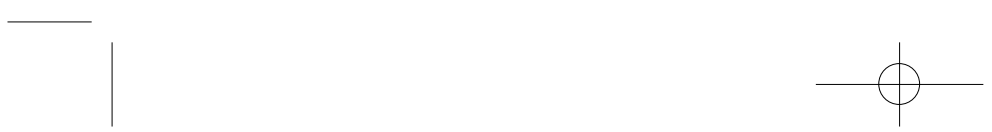